\title{
SPECTRAL PROPERTIES OF ELEMENTS IN DIFFERENT BANACH ALGEBRAS
}

\author{
by J. J. GROBLER and H. RAUBENHEIMER
}

(Received 7 June, 1989)

1. Introduction. Let $A$ be a Banach algebra with unit 1 and let $B$ be a Banach algebra which is a subalgebra of $A$ and which contains 1. In [5] Barnes gave sufficient conditions for $B$ to be inverse closed in $A$. In this paper we consider single elements and study the question of how the spectrum relative to $B$ of an element in $B$ relates to the spectrum of the element relative to $A$.

This question has been studied by a number of authors (see $[14,2,3,4,12,13,15]$ ) under various conditions, usually requiring the norm on $B$ to be finer than the norm on $A$ and in some cases requiring $B$ to be a Banach algebra of operators on a Banach lattice. We shall merely assume the algebra $B$ to be a subalgebra of $A$ and we shall not assume any relationship between the norms of $A$ and $B$. Of course, if $B$ is semi-simple and closed in $A$, the embedding of $B$ into $A$ is continuous (see [7, Theorem 25.9]). We do not intend to enter into a discussion of the question whether or not this is always true-this is a deep question which seems also to be related to the model of set theory used (see [8]). The point we want to emphasize is that the main results are independent of the continuity of the embedding.

If $A$ is an algebra we denote the spectrum of an element $a \in A$ by $\sigma(a, A)$ and its spectral radius by $r_{A}(a)$. In Section 2 we investigate the relationship between the spectra $\sigma(b, B)$ and $\sigma(b, A)$ for an element $b \in B \subset A$. This investigation is continued in Sections 3 and 5 where essential and Weyl spectra are also involved. In Section 4 we study the behaviour of the spectra under perturbation by Riesz elements. In Section 6 we solve a problem posed in the paper [4] which does not involve two different algebras, but the solution of which uses some of the ideas developed in this paper.

2. The relationship between $\sigma(b, B)$ and $\sigma(b, A)$. Our first result relates idempotents in different algebras and is basic to our considerations. It is a generalisation of a result due to $\mathrm{H}$. $\mathrm{H}$. Schaefer [14] and W. Arendt [2].

2.1 Proposition. Let $A$ and $B$ be Banach algebras and let $\psi: B \rightarrow A$ be $a$ multiplicative linear map with $\psi(1)=1$. Let $D$ be a clopen set in both $\sigma(b, B)$ and $\sigma(\psi(b), A)$, and let

$$
e_{B}:=\frac{1}{2 \pi i} \int_{\Gamma}(\lambda-b)^{-1} d \lambda \text { and } f_{A}:=\frac{1}{2 \pi i} \int_{\Gamma}(\lambda-\psi(b))^{-1} d \lambda
$$

with $\Gamma$ a rectifiable curve surrounding $D$ in $\mathbb{C} \backslash \sigma(b, B)$. Then $\psi\left(e_{B}\right)=f_{A}$.

Proof. Let $C_{B}$ and $C_{A}$ be maximal commutative subalgebras of $B$ and $A$ which respectively contains $b$ and $\psi(b)$ and satisfy $\psi\left(C_{B}\right) \subset C_{A}$. If $\phi$ is a multiplicative linear functional on $C_{A}$, then $\phi \circ \psi$ is multiplicative (and hence continuous) on $C_{B}$. Therefore,

$$
\phi \circ \psi\left(e_{B}\right)=\frac{1}{2 \pi i} \int_{\Gamma} \phi \circ \psi\left[(\lambda-b)^{-1}\right] d \lambda=\frac{1}{2 \pi i} \int_{\Gamma} \phi\left[(\lambda-\psi(b))^{-1}\right] d \lambda=\phi\left(f_{A}\right) .
$$

Glasgow Math. J. 33 (1991) 11-20. 
Now, $\psi\left(e_{B}\right)$ is idempotent and from the calculation above we find that $\psi\left(e_{B}\right)-\psi\left(e_{B}\right) f_{A}$ and $f_{A}-\psi\left(e_{B}\right) f_{A}$ are both idempotents belonging to the radical of $C_{A}$. Hence they are both zero, showing that $\psi\left(e_{B}\right)=\psi\left(e_{B}\right) f_{A}=f_{A}$.

2.2 Corollary. Let $A$ and $B$ be Banach algebras such that $B \subset A$ and such that $1 \in B$. Let $D$ be a clopen set in both $\sigma(b, B)$ and $\sigma(b, A)$, and let $\Gamma$ be a rectifiable curve which surrounds $D$ in $\mathbb{C} \backslash \sigma(b, B)$. If

$$
e_{B}=\frac{1}{2 \pi i} \int_{\Gamma}(\lambda-b)^{-1} d \lambda \text { and } e_{A}=\frac{1}{2 \pi i} \int_{\Gamma}(\lambda-b)^{-1} d \lambda
$$

are defined as elements of $B$ and $A$ respectively, then $e_{B}=e_{A}$.

Our next result contains [14 Proposition 4.1] and [2 Proposition 2.2].

2.3 Corollary. Let $A$ and $B$ be Banach algebras such that $B \subset A$ and such that $1 \in B$. If $b \in B$ and if $D$ is a non-void clopen subset of $\sigma(b, B)$, then $D \cap \sigma(b, A) \neq \varnothing$.

Proof. Let $b \in B$ and let $D$ be a clopen subset of $\sigma(b, B)$. Suppose that $D \cap$ $\sigma(b, A)=\varnothing$ and let $\Gamma$ be a closed rectifiable curve in $\mathbb{C} \backslash \sigma(b, B)$ which surrounds $D$ and separates it from $\sigma(b, A)$. Then with $e_{B}$ and $e_{A}$ defined as in $2.2, e_{B} \neq 0$ and $e_{A}=0$, a contradiction.

If $K$ is a subset of $\mathbb{C}$ we denote its set of isolated points by iso $K$.

2.4 Corollary. (a) The set iso $\sigma(b, B) \subset \sigma(b, A)$ and hence iso $(\sigma(b, B) \backslash \sigma(b, A))=\varnothing$.

(b) If $\sigma(b, B) \backslash \sigma(b, A) \neq \varnothing$, then it is uncountable.

(c) If $\sigma(b, B)$ is totally disconnected, then $\sigma(b, B)=\sigma(b, A)$.

We refer the reader to [4] for examples which seem to indicate that these results are the best we can expect. In example (A1) of [4] an element $b$ is exhibited for which the spectral radius $r_{A}(b)=1$ and $r_{B}(b) \geq \sqrt{2}$. This illustrates that $\partial \sigma(b, B) \notin \sigma(b, A)$. Even if $r_{A}(b)=r_{B}(b)$, Example (A2) of [4] shows that $\partial \sigma(b, B)$ need not be contained in $\sigma(b, A)$.

We recall that a two-sided ideal of a Banach algebra $B$ is called inessential whenever the spectrum of every element in the ideal is either finite or a sequence converging to zero. If $J$ is an inessential ideal of $B$, then the closure of $J$ in $B$, denoted by $J_{B}$, is also an inessential ideal in $B$ ([1, Corollary 2.6]). An element $b \in B$ is called a Riesz element relative to the closed ideal $J_{B}$ if the spectrum of the element $b+J_{B}$ in the quotient algebra $B / J_{B}$ consists of zero. We denote the set of Riesz elements in $B$ relative to $J_{B}$ by $\mathscr{R}\left(B, J_{B}\right)$. For the properties of these elements we refer to [6, Section R.1].

2.5 Corollary. ([2 Theorem 2.6], [12 Theorem 4]). Let $A$ and $B$ be Banach algebras such that $B \subset A$ and such that $1 \in B$. Let $J$ be a two-sided inessential ideal of $B$; then $\sigma(b, B)=\sigma(b, A)$ for all $b \in \mathscr{R}\left(B, J_{B}\right)$.

Proof. If $b$ is a Riesz element in $B$ relative to $J_{B}$, then by [6, Theorem R.2.6] or [1, Corollary 2.5], $\sigma(b, B)$ is either finite or a sequence converging to zero; the result follows from 2.4 .

2.6 Corollary. Let $A$ and $B$ be Banach algebras such that $B \subset A$ and such that $1 \in B$. Let $J$ be a two-sided inessential ideal of $B$, and suppose that $f$ is an analytic function 
which is defined on a neighbourhood $V$ of $\sigma(b, B)$ and which is non-constant on every component of $V$. Then $\sigma(b, B)=\sigma(b, A)$ if either (a) or (b) holds.

(a) $f(b) \in \mathscr{R}\left(B, J_{B}\right)$.

(b) $\sigma(b, A)$ is countable and $\sigma(f(b), B)=\sigma(f(b), A)$.

Proof. In both cases we claim that $f[\sigma(b, B)]$ is at most countable; if (a) holds it follows from the fact that $f(b) \in \mathscr{R}\left(B, J_{B}\right)$ and $f[\sigma(b, B)]=\sigma(f(b), B)$, and if (b) holds it is obvious. For any given $\mu \in \mathbb{C}$ and any component $U$ of $V$, there exists at most a finite number of elements $\lambda \in U \cap \sigma(b, B)$ such that $f(\lambda)=\mu$, since $f$ is non-constant and $\sigma(b, B)$ is bounded. By the compactness of $\sigma(b, B)$ we conclude that $\{\lambda \in \sigma(b, B): f(\lambda)=$ $\mu\}$ is finite for every $\mu \in f[\sigma(b, B)]$. Hence, $\sigma(b, B)$ is at most countable.

We note that in general the countability of $\sigma(b, A)$ is not sufficient for the two spectra $\sigma(b, A)$ and $\sigma(b, B)$ to be equal (see [2, Example 3.7]. Part (b) was proved in the special case of operator algebras in [4, Theorem 7.3].

As a final application of 2.1 we show that a result of B. A. Barnes ([5, Theorem 1]) also derives from it.

2.7 Theorem (B. A. Barnes). Let $A$ and $B$ be Banach algebras such that $B$ is $a$ commutative subalgebra of $A$ and such that $1 \in B$. Denote by $\Omega_{B}$ the space of non-zero multiplicative linear functionals on $B$ equipped with the B-topology. If $\Omega_{B}$ is totally disconnected then, for every $b \in B$, we have $\sigma(b, B)=\sigma(b, A)$.

Proof. We note first of all that we may assume without loss of generality that $A$ is also commutative, for else we consider instead a maximal commutative subalgebra of $A$ which contains $B$. Let $b \in B$ and suppose that there exists a number $\lambda \in \sigma(b, B) \backslash \sigma(b, A)$. Then there exists an element $\phi \in \Omega_{B}$ such that $\phi(b)=\lambda$, i.e., $b(\phi)=\lambda$. Assume now that $U(\lambda, \varepsilon)$ is an open disc in $\mathbb{C} \backslash \sigma(b, A)$ centered at $\lambda$. Then some open neighbourhood $V \subset \Omega_{B}$ of $\phi$ is mapped by $\bar{b}$ into $U(\lambda, \varepsilon)$. If we denote by $\Delta$ the set of all restrictions to $B$ of elements of $\Omega_{A}$, it follows from $U(\lambda, \varepsilon) \subset \mathbb{C} \backslash \sigma(b, B)$ that $V \cap \Delta=\varnothing$. Our assumption that $\Omega_{B}$ is totally disconnected implies now that $\phi \in D$ for some clopen subset $D \subset V$, (see [11, Theorem 2.14]). By the Shilov idempotent theorem ([7, Theorem 21.5]) there exists an element $e \in B$ such that $\hat{e}=\chi_{D}$, with $\chi_{D}$ the characteristic function of $D$. Then $\sigma(e, B)=\{0,1\}$, and $\sigma(e, A)=\{0\}$. This contradiction to 2.4 concludes the proof.

3. Essential spectra. In this section we look at spectra of quotient algebras. In order to prove the analogue of 2.3 in this setting, we first prove the following lemma.

3.1 Lemma. Let $A$ and $B$ be Banach algebras such that $B \subset A$ and such that $1 \in B$. Let $J$ be a two-sided inessential ideal of $A$ such that $J \cap B$ is a two-sided inessential ideal in $B$. If $b \in B \cap \mathscr{R}\left(A, J_{A}\right)$ then we have

(a) $b \in(J \cap B)_{B}$ if and only if $b^{2}-b \in(J \cap B)_{B}$,

(b) $b \in \mathscr{R}\left(B,(J \cap B)_{B}\right)$ if and only if $b^{2}-b \in \mathscr{R}\left(B,(J \cap B)_{B}\right)$.

Proof. In one direction there is nothing to prove. In the converse direction we prove (a) and (b) simultaneously by putting $X$ equal to either $(J \cap B)_{B}$ or to $\mathscr{R}\left(B,(J \cap B)_{B}\right)$ and assuming that $b^{2}-b \in X \subset \mathscr{R}\left(B,(J \cap B)_{B}\right)$. By 2.6, $\sigma=\sigma(b, B)=\sigma(b, A)$. We claim that there exists an element $c \in J \cap B$ such that $1-b-c$ is invertible in $A$. If $1 \notin \sigma$, choose $c=0$; if $1 \in \sigma$, note that since $b \in \mathscr{R}\left(A, J_{A}\right)$, we have that 1 is an isolated point of $\sigma$. 
Therefore, if $\Gamma$ is a small circle isolating 1 in $\sigma$ the idempotent $e=\frac{1}{2 \pi i} \int_{\Gamma}(\lambda-b)^{-1} d \lambda$ is an element of $B$, but since $b \in \mathscr{R}\left(A, J_{A}\right)$ is also an element of $J$ (see [6, Theorem R.2.5] or [1, Corollary 2.5] and apply 2.2). But $1 \notin \sigma(b-b e, A)$ and so we put $c$ equal to $-b e \in J \cap B$. If we define $s:=b+c$, then since $b c=c b$,

$$
s^{2}-s=\left(b^{2}-b\right)+\left(2 b c+c^{2}-c\right) \in X+(J \cap B) \subset X .
$$

Again 2.6 applies and we get $\sigma(s, B)=\sigma(s, A)$ and because $1-s=1-b-c$ is invertible in $A$, it is also invertible in $B$. But then

$$
s=(1-s)^{-1}\left(s-s^{2}\right)=\left(s-s^{2}\right)(1-s)^{-1} \in X,
$$

(by the well known properties of Riesz elements if $X=\mathscr{R}\left(B,(J \cap B)_{B}\right)$ (see [6, Theorem R.2(iii)]). Therefore, $b=s-c \in X-(J \cap B) \subset X$.

3.2 Theorem. Let $A$ and $B$ be Banach algebras such that $B \subset A$ and such that $1 \in B$. Let $J$ be a two-sided inessential ideal of $A$ such that $J \cap B$ is a two-sided inessential ideal in $B$ and suppose that $(J \cap B)_{B} \subset J_{A}$. If $b \in B$ and if $D$ is a non-void clopen subset of $\sigma\left(J+(J \cap B)_{B}, B /(J \cap B)_{B}\right)$, then $D \cap \sigma\left(b+J_{A}, A / J_{A}\right) \neq \varnothing$.

Proof. The mapping $\psi: B /(J \cap B)_{B} \rightarrow A / J_{A}$ given by $\psi\left(b+(J \cap B)_{B}\right):=b+J_{A}$, $b \in B$, is a well-defined algebra homomorphism which maps the unit onto the unit. Let $\Gamma$ be a closed rectifiable curve in $\mathbb{C} \backslash \sigma\left(b+(J \cap B)_{B}, B /(J \cap B)_{B}\right)$ that surrounds $D$ and separates it from $\sigma\left(b+J_{A}, A / J_{A}\right)$ and let

$$
\tilde{p}_{B}:=\frac{1}{2 \pi i} \int_{\Gamma}\left(\lambda-\left(b+(J \cap B)_{B}\right)\right)^{-1} d \lambda
$$

Then $\tilde{p}_{B} \neq 0$. If we write $\tilde{p}_{B}=p+(J \cap B)_{B}$, with $p \in B$, then it follows from $\left(\tilde{p}_{B}\right)^{2}=\tilde{p}_{B}$ that $p^{2}-p \in(J \cap B)_{B}$. By Proposition 2.1,

$$
\psi\left(\tilde{p}_{B}\right)=\frac{1}{2 \pi i} \int_{\Gamma}\left(\lambda-\left(b+J_{A}\right)\right)^{-1} d \lambda=0
$$

and hence $p \in J_{A}$. By Lemma 3.1(a) we get $p \in(J \cap B)_{B}$ which contradicts the fact that $\tilde{p}_{B} \neq 0$.

We note that the condition $(J \cap B)_{B} \subset J_{A}$ in the theorem is automatically satisfied if $B$ has a finer norm than $A$. If $E$ is a Banach lattice and if we denote by $L^{r}(E)$ the set of regular operators on $E$ equipped with the regular norm, (see [2]), then $L^{r}(E)$ is a Banach algebra contained in the Banach algebra $L(E)$ of all bounded linear operators on $E$ and the regular norm is finer than the operator norm. It was for these two algebras that Theorem 3.2 was first proven by Arendt and Sourour in [4, Theorem 6.2]. Here $J$ is the ideal of finite rank operators and its closure in the regular norm is denoted by $K^{r}$, an inessential ideal.

Of course, the same corollaries derived from 2.3 can now be stated for this case. Also, by taking $J$ to be the zero ideal, we regain 2.3 .

Our next result shows that if $J$ is an inessential ideal in both $A$ and in $B$, there is a close connection between the essential spectrum $\sigma(b+J, B / J)$, the spectrum $\sigma(b, B)$ and the spectrum $\sigma(b, A)$. 
3.3 Lemma. Let $A$ and $B$ be Banach algebras such that $B \subset A$ and such that $1 \in B$. Let $J$ be a two-sided inessential ideal both in $A$ and in $B$. If $d \in \mathscr{R}\left(B, J_{B}\right)$ and if $a \in A$ satisfies $a(1-d) \in B$, then $a \in B$.

Proof. As in the proof of Lemma 3.1 we can prove the existence of an element $c \in J$ such that $1-d-c$ is invertible in $B$. (If $1 \notin \sigma(d, B)$ choose $c=0$, and if $1 \in \sigma(d, B)$, choose $c=-d e$, with $e=\frac{1}{2 \pi i} \int_{\Gamma}(\lambda-d)^{-1} d \lambda$, where $\Gamma$ is a small circle with centre 1 .) Since $J$ is an ideal in $A$ also, $a c \in J \subset B$. The equality $a=(a(1-d)-a c)(1-d-c)^{-1}$ shows that $a \in B$.

3.4 Theorem. Let $A$ and $B$ be Banach algebras such that $B \subset A$ and such that $1 \in B$. Let $J$ be a two-sided inessential ideal in $A$ and in $B$. Then, for every $b \in B$,

$$
\sigma(b, B)=\sigma(b, A) \cup \sigma\left(b+J_{B}, B / J_{B}\right) .
$$

Proof. For the non-trivial inclusion assume that $\lambda \notin \sigma\left(b+J_{B}, B / J_{B}\right)$ and that $\lambda \notin \sigma(b, A)$. Then $(\lambda-b)^{-1}$ exists in $A$ and there exist elements $c \in B$ and $d \in J_{B}$ such that $(\lambda-b) c=1+d$. From this we get

$$
(\lambda-b)^{-1}(1+d)=c \in B
$$

and it follows from the preceding lemma that $\lambda \notin \sigma(b, B)$.

3.5 Corollary. Let $A$ and $B$ be Banach algebras such that $B \subset A$ and such that $1 \in B$. Let $J$ be a two-sided inessential ideal in $A$ and in $B$. Then, for every $b \in B$ we have

(a) $\sigma(b, B) \backslash \sigma\left(b+J_{B}, B / J_{B}\right) \subset \sigma(b, A) \backslash \sigma\left(b+J_{A}, A / J_{A}\right)$, whenever $J_{B} \subset J_{A}$;

(b) $\sigma(b, B)=\sigma(b, A)$, whenever $\sigma\left(b+J_{B}, B / J_{B}\right)=\sigma\left(b+J_{A}, A / J_{A}\right)$.

Examples 5.1 and 5.2 of [4] show that the reverse inclusion in (a) and the reverse implication in (b) are false.

For arbitrary proper ideals (not necessarily closed or inessential), a weaker analogue of Theorem 3.4 holds.

3.6 Theorem. Let $A$ and $B$ be Banach algebras such that $B \subset A$ and such that $1 \in B$. Let $J$ be a proper two-sided ideal in $A$ and in $B$. Then, for every $b \in B$,

(a) $\sigma(b, B)=\sigma(b, A) \cup \sigma(b+J, B / J)$;

(b) $\sigma(b, B)=\sigma(b, A)$, whenever $b^{k} \in J$ for some $k \in \mathbb{N}$.

Proof. (a) If $\lambda \notin \sigma(b+J, B / J)$ and $\lambda \notin \sigma(b, A)$, there exist elements $c \in B$ and $d \in J$ such that $(\lambda-b) c=1+d$ and so $(\lambda-b)^{-1}=c-(\lambda-b)^{-1} d \in B$.

(b) If $b^{k} \in J$, we have $\sigma\left(b^{k}+J, B / J\right)=\{0\}$ and by the spectral theorem for polynomials (see $[7,1.5 .5]) \sigma(b+J, B / J)=\{0\}$. But, by Corollary $2.4(\mathrm{a}), 0$ cannot be the only element of $\sigma(b, B)$ which does not belong $\sigma(b, A)$ and so (b) holds.

We may remark that it is very easy to verify directly that $3.6(\mathrm{~b})$ holds for $J$ either a left or a right ideal, without looking at the quotient algebra. One can also derive this from the above theorem by considering a maximal commutative subalgebra $C_{B}$ of $B$ which contains the element $b$. In this case $J \cap C_{B}$ is a two-sided ideal in $C_{B}$ and an application of 3.6(b) yields the required result. These results were proved in references [13] and in [14] for the algebras $A=L(E)$ and $B=L^{r}(E)$ with $E$ a Banach lattice and $J$ being either the cone of absolutely summing or the majorizing operators on $E$. 
4. Perturbation results. In this section we study the behaviour of the spectra under perturbation by Riesz elements. Our first result is a consequence of Corollary 2.5 and was proved in the order theoretic setting in [13]. (See also [4, Theorem 2.1].)

4.1 Theorem. (Perturbation by Riesz elements). Let $A$ and $B$ be Banach algebras such that $B \subset A$ and such that $1 \in B$. Let $J$ be a two-sided inessential ideal in $B$. If $a \in B$ and $b \in \mathscr{R}\left(B, J_{B}\right)$ satisfy either $a b=b a$ or $b \in J_{B}$, then $\sigma(a, B) \backslash \sigma(a+b, B) \subset \sigma(a, A)$.

Proof. Let $\lambda \in \sigma(a, B) \backslash \sigma(a+b, B)$. Since $\lambda-a=(\lambda-a-b)\left(1+(\lambda-a-b)^{-1} b\right)$ we have that $1 \in \sigma\left(-(\lambda-a-b)^{-1} b, B\right)$. But, since $b \in \mathscr{R}\left(B, J_{B}\right)$ and $(\lambda-a-b)^{-1} \in B$ we have in the case that $a b=b a$ (which implies $(\lambda-a-b)^{-1} b=b(\lambda-a-b)^{-1}$ ) that $-(\lambda-a-b)^{-1} b \in \mathscr{R}\left(B, J_{B}\right)$. Also in the case that $b \in J_{B}$ we get $-(\lambda-a-b)^{-1} b \in J_{B} \subset$ $\mathscr{R}\left(B, J_{B}\right)$. Hence, by Corollary $2.5, \sigma\left(-(\lambda-a-b)^{-1} b, B\right)=\sigma\left(-(\lambda-a-b)^{-1} b, A\right)$. Therefore, $1 \in \sigma\left(-(\lambda-a-b)^{-1} b, A\right)$ and so $\lambda \in \sigma(a, A)$.

4.2 Corollary. If $a \in B$ and $b \in \mathscr{R}\left(B, J_{B}\right)$, and either $a b=b a$ or $b \in J_{B}$, then

(a) $\sigma(a+b, B) \backslash \sigma(a, B) \subset \sigma(a+b, A) \backslash \sigma(a, A)$;

(b) $\sigma(a+b, B) \backslash \sigma(a, B)=\sigma(a+b, A) \backslash \sigma(a, A)$, whenever $\sigma(a, B)=\sigma(b, A)$.

The inclusion in (a) can be proper but we do not know whether or not the commutativity condition can be removed.

Let $J$ be a two-sided inessential ideal in a Banach algebra $A$. If $\lambda$ is an isolated point of the spectrum of an element $\boldsymbol{a}$ in $A$ we denote the idempotent

$$
\frac{1}{2 \pi i} \int_{\Gamma}(\xi-a)^{-1} d \xi
$$

with $\Gamma$ a circle around $\lambda$ which does not contain any other points of $\sigma(a, A)$, by $e(\lambda, a)$. Following [1] we define for an element $a \in A$ the set $D(a, A, J)$ as follows:

$$
\lambda \notin D(a, A, J) \Leftrightarrow\left\{\begin{array}{l}
\lambda \notin \sigma(a, A) \\
\text { or } \\
\lambda \text { is isolated in } \sigma(a, A) \text { and } e(\lambda, a) \in J .
\end{array}\right.
$$

The set $D(a, A, J)$ is compact and $\sigma(a, A) \backslash D(a, A, J)$ is discrete and hence countable. Moreover, if $A$ and $B$ are Banach algebras with $B \subset A$ a subalgebra and $1 \in B$ and if $J$ is an inessential ideal in $A$ such that $J \cap B$ is inessential in $B$ then it is easily seen that, for $b \in B, \sigma(b, A)=\sigma(b, B)$ if and only if $D(b, B, J \cap B)=D(b, A, J)$.

Let $K$ be a compact subset of the complex plane. We define the polynomially convex hull $K^{\wedge}$ of $K$ as usual by

$$
K^{\wedge}:=\left\{z \in \mathbb{C}:|p(z)| \leq\|p\|_{K} \text { for every polynomial } p\right\},
$$

where $\|p\|_{K}:=\sup \{|p(z)|: z \in K\}$. The set $K$ is called polynomially convex if $K=K^{\wedge}$.

Our second perturbation result is based on a theorem which was proved by $B$. Aupetit [1, Theorem 2.4] and which was extended in [9].

4.3 Theorem. Let $A$ and $B$ be Banach algebras with $B \subset A$ a subalgebra and $1 \in B$. Suppose that $J$ is a two-sided inessential ideal in $A$ such that $J \cap B$ is a two-sided inessential ideal in $B$. If $a \in B$ and if $b \in \mathscr{R}\left(A, J_{A}\right) \cap \mathscr{R}\left(B,(J \cap B)_{B}\right)$ satisfies either $a b=b a$ or $b \in J_{A} \cap(J \cap B)_{B}$, then $\sigma(a+b, B)=\sigma(a+b, A)$ whenever $\sigma(a, B)=\sigma(a, A)$ and $\sigma(a, B)$ satisfies $\partial \sigma(a, B)=\sigma(a, B)^{\wedge}$. 
Proof. It follows from [1, Theorem 2.4] and [9, Theorem 2] that $\mathbb{C} \backslash D(a, B, J \cap B)$ and $\mathbb{C} \backslash D(a+b, B, J \cap B)$ have the same unbounded components. Therefore, $D(a, B, J \cap B)^{\wedge}=D(a+b, B, J \cap B)^{\wedge}$. From the definition of the set $D(a, B, J \cap B)$ we get that $\partial D(a, B, J \cap B)=D(a, B, J \cap B)^{\wedge}$ is also satisfied. From this and

$$
\partial D(a, B, J \cap B) \subset D(a, B, J \cap B) \subset D(a, B, J \cap B)^{\wedge}=\partial D(a, B, J \cap B)
$$

we derive that $D(a, B, J \cap B)=D(a, B, J \cap B)^{\wedge}$ and consequently that $D(a, B, J \cap B)=$ $D(a+b, B, J \cap B)^{\wedge}$. If $U$ is a bounded component of $\mathbb{C} \backslash D(a+b, B, J \cap B)$, then

$$
U \subset D(a+b, B, J \cap B)^{\wedge}=D(a, B, J \cap B)=\partial D(a, B, J \cap B) .
$$

Since the latter set has empty interior, $U=\varnothing$. Hence, $D(a+b, B, J \cap B)=$ $D(a+b, B, J \cap B)^{\wedge}$ and it follows that $D(a+b, B, J \cap B)=D(a, B, J \cap B)$. The same argument applies to show that also $D(a+b, A, J)=D(a, A, J)$. It follows from $\sigma(a, B)=\sigma(a, A)$ that $D(a, B, J \cap B)=D(a, A, J)$ and therefore $D(a+b, B, J \cap B)=$ $D(a+b, A, J)$. From our remark preceding 4.3 we deduce that $\sigma(a+b, B)=$ $\sigma(a+b, A)$.

Of course, two obvious instances of the above theorem are when $\sigma(a, B)=\sigma(a, A)$ and $\sigma(a, B)$ is either real or countable.

5. Weyl spectra. Let $A$ be a Banach algebra and let $J$ be a closed two-sided ideal in A. The set

$$
\omega(a, A, J):=\bigcap\{\sigma(a+c, A): c \in J\}
$$

is called the Weyl spectrum of $a$ in $A$ relative to $J$. It is well known (see [10, Theorem 2.3]) that

$$
\sigma(a+J, A / J) \subset \omega(a, A, J) \subset \sigma(a, A)
$$

and if $J$ is an ideal in $A$ such that $(J \cap B)_{B} \subset J_{A}$ with $B \subset A$ a subalgebra of $A$, then $\omega\left(b, A, J_{A}\right) \subset \omega\left(b, B,(J \cap B)_{B}\right)$.

If $J$ is a two-sided inessential ideal in $A$ then by [10, Theorem 1]

$$
D\left(b, A, J_{A}\right)=\bigcap\left\{\sigma(b+c, A): c \in J_{A} \text { and } b c=c b\right\}
$$

and so

$$
\sigma\left(b+J_{A}, A / J_{A}\right) \subset \omega\left(b, A, J_{A}\right) \subset D\left(b, A, J_{A}\right) \subset \sigma(b, A) .
$$

A reformulation of Theorem 4.1 yields the following result which was proved in the order theoretic case in [4, Theorem 4.4].

5.1 THEOREM. Let $A$ and $B$ be Banach algebras such that $B \subset A$ and such that $1 \in B$. Let $J$ be a two-sided inessential ideal in $B$. For arbitrary $a \in B$ we have $\sigma(a, B)=$ $\sigma(a, A) \cup \omega\left(a, B, J_{B}\right)$.

From the remarks preceding the theorem the following corollary is clear.

5.2 Corollary. Let $A$ and $B$ be as in 5.1 and let $J$ be a two-sided ideal in $A$ such that $J \cap B$ is also inessential in $B$ and such that $(J \cap B)_{B} \subset J_{A}$. For arbitrary $a \in B$ we have

$$
\sigma(a, B) \backslash \omega\left(a, B,(J \cap B)_{B}\right) \subset \sigma(a, A) \backslash \omega\left(a, A, J_{A}\right) .
$$


The reverse inclusion is false in general as can be seen from [4, Example 5.2]. In order to prove our final result in this section we need the following lemma.

5.3 Lemma. If $A$ is a Banach algebra and if $J$ is a two-sided inessential ideal in $A$, then for arbitrary $a \in A$,

$$
\omega\left(a, A, J_{A}\right)=\bigcap\{\sigma(a+b, A): b \in J\} .
$$

Proof. For the non-trivial inclusion assume that $\lambda \notin \omega\left(a, A, J_{A}\right)$. Then there exist $b \in J_{A}$ and an invertible element $c \in A$ such that $\lambda-a=c+b$. Therefore, $\lambda-a=$ $c\left(1+c^{-1} b\right)$. Since $c^{-1} b \in J_{A}$ and $J_{A}$ is inessential, there exists an element $p \in J$ such that $1+c^{-1} b-p$ is invertible in $A$ (see the proof of 3.1 and 3.3). This shows that $\lambda-a-c p=c\left(1+c^{-1} b-p\right)$ is invertible in $A$, with $c p \in J$. Therefore,

$$
\lambda \notin \bigcap\{\sigma(a+b, A): b \in J\} .
$$

This completes the proof.

We list some of the known relationships between the notions studied thus far.

5.4 Theorem. Let $A$ and $B$ be Banach algebras such that $B \subset A$ is a subalgebra of $A$ and such that $1 \in B$. Suppose that $J$ is a two-sided inessential ideal both in $A$ and in $B$ such that $J_{B} \subset J_{A}$. For arbitrary $a \in B$ consider the following statements.

(a) $\sigma\left(a+J_{A}, A / J_{A}\right)=\sigma\left(a+J_{B}, B / J_{B}\right)$.

(b) $\omega\left(a, A, J_{A}\right)=\omega\left(a, B, J_{B}\right)$.

(c) $\sigma(a+b, A)=\sigma(a+b, B)$ for every $b \in J_{B}$.

(d) $\sigma(a, A)=\sigma(a, B)$.

(e) $D(a, A, J)=D(a, B, J)$.

Then the following implications are valid:

(a) $\Rightarrow(\mathrm{b}) \Leftrightarrow(\mathrm{c}) \Rightarrow(\mathrm{d}) \Leftrightarrow(\mathrm{e})$.

Proof. The implication (a) $\Rightarrow$ (c) follows from 3.5 and the facts that $J_{B} \subset J_{A}$ and that the essential spectra $\sigma\left(., A / J_{A}\right)$ and $\sigma\left(., B / J_{B}\right)$ are stable under perturbation by elements in $J_{B}$. The implication (c) $\Rightarrow$ (b) follows from 5.3. In order to prove that (b) $\Rightarrow$ (c), assume that $\omega\left(a, A, J_{A}\right)=\omega\left(a, B, J_{B}\right)$. By 5.1 we have $\sigma(a, B)=\sigma(a, A) \cup \omega\left(a, B, J_{B}\right)$ and hence $\sigma(a, B)=\sigma(a, A) \cup \omega\left(a, A, J_{A}\right) \subset \sigma(a, A)$. Therefore, $\sigma(a, A)=\sigma(a, B)$. Applying the above argument to $a+b$ and noting that $\omega\left(a+b, B, J_{B}\right)=\omega\left(a, B, J_{B}\right)$, for all $b \in J_{B}$, we get (c). That (d) and (e) are equivalent is obvious.

The implications (b) $\Rightarrow(\mathrm{a})$ and $(\mathrm{d}) \Rightarrow$ (c) are false (see [4, Examples 5.1 and 5.2]).

6. The pure spectrum. In this section we characterise $\sigma(a, A) \backslash \sigma\left(a+J_{A}, A / J_{A}\right)$, where $A$ is a Banach algebra and $J$ an inessential two-sided ideal in $A$. We call an isolated point $\lambda \in \sigma(a, A)$ a $J$-projection point if the projection $e(\lambda, a)$ associated with $a$ and $\lambda$ is an element of $J$. We shall also denote the set of $J$-Fredholm elements of $A$ by $\Phi_{J}(A)$ and the index of an element $c \in \Phi_{J}(A)$ by ind $(c)$ (see [6, Definition F.3.12 and F.3.6]).

6.1 Theorem. Let $J$ be a two-sided inessential ideal in the Banach algebra $A$. Then for every $a \in A$ the set $\sigma(a, A) \backslash \sigma\left(a+J_{A}, A / J_{A}\right)$ is the union of $J$-projection points and of some of the holes of $\sigma\left(a+J_{A}, A / J_{A}\right)$. It includes every hole of $\sigma\left(a+J_{A}, A / J_{A}\right)$ in which $\operatorname{ind}(\lambda-a) \neq 0$. 
Proof. Suppose that $\lambda \in \sigma(a, A) \backslash \sigma\left(a+J_{A}, A / J_{A}\right)$. If $\lambda$ is isolated in $\sigma(a, A)$ let $\Gamma$ be a circle centred at $\lambda$ and separating $\lambda$ from the rest of the spectrum and denote the quotient map of $A$ onto $A / J_{A}$ by $\phi$. By Theorem 1.1, we have $\phi(e(\lambda, a))=e\left(\lambda, a+J_{A}\right)$. But, since $\lambda \notin \sigma\left(a+J_{A}, A / J_{A}\right)$ the latter projection is the zero projection, i.e., $e=e(\lambda, a) \in J_{A}$. Now, $e J_{A} e$ is a closed subalgebra of $A$ and hence a Banach algebra with unit $e$, in which $e J e$ is a dense two-sided ideal; therefore, $e J e=e J_{A} e$. Consequently, $e=e^{3} \in e J_{A} e=e J e \subset J$, and we have shown that $\lambda$ is a $J$-projection point. If $\lambda$ is not isolated, then

$$
\lambda \in D(\lambda, a, A) \subset D(\lambda, a, A)^{\wedge} \subset \sigma\left(a+J_{A}, A / J_{A}\right)^{\wedge},
$$

where the last inclusion follows from [1, Theorem 2.4(iii)]. Hence, $\lambda$ belongs to a hole in $\sigma\left(a+J_{A}, A / J_{A}\right)$. Suppose now that $\lambda \in \mathbb{C} \backslash \sigma\left(a+J_{A}, A / J_{A}\right)$ belongs to a hole of $\sigma\left(a+J_{A}, A / J_{A}\right)$ and that ind $(\lambda-a) \neq 0$; then $\lambda-a$ cannot be invertible ([6, F.3.7]) and so every element in this hole belongs to $\sigma(a, A) \backslash \sigma\left(a+J_{A}, A / J_{A}\right)$. This completes the proof.

6.2 Corollary. Every point in $\sigma(a, A)$ which belongs to the unbounded component of $\mathbb{C} \backslash \sigma\left(a+J_{A}, A / J_{A}\right)$ is isolated and is therefore a J-projection point.

Corollary 6.2 is a generalization of [4, Corollary 3.8]. Theorem 6.1 provides the answer to the question posed in [4]. We state explicitly the theorem for the case considered there.

Let $E$ be a Banach lattice and let $L^{\prime}(E)$ denote the Banach algebra of regular operators on $E$ equipped with the regular norm. If $J$ is the closure in $r$-norm of the ideal of finite rank operators in $L^{r}(E)$ the spectrum $\sigma\left(T+J, L^{r}(E) / J\right)$ is denoted by $\sigma_{o e}(T)$ and the spectrum $\sigma\left(T, L^{r}(E)\right)$ by $\sigma_{o}(T)$. In the next theorem the index of an operator will have its usual meaning.

6.3 Theorem. Let $E$ be a Banach lattice and let $T \in L^{r}(E)$. Then $\sigma_{0}(T) \backslash \sigma_{o e}(T)$ is the union of isolated points with pertaining projections of finite rank together with some of the holes of $\sigma_{o e}(T)$. Moreover, $\sigma_{o}(T) \backslash \sigma_{o e}(T)$ contains all the holes of $\sigma_{o e}(T)$ in which the index of the operator $\lambda-T$ is non-zero.

Proof. The first part follows from the abstract Theorem 6.1. If $\lambda \in \mathbb{C} \backslash \sigma_{o e}(T)$ belongs to a hole in $\sigma_{o e}(T)$, and if the index of $\lambda-T$ is non-zero, then $\lambda-T$ cannot be invertible in $L^{r}(E)$ because if it were, it would have been invertible in $L(E)$ implying that it has index zero. Hence, $\lambda \in \sigma_{o}(T)$. It follows that every hole of $\sigma_{o e}(T)$ in which the index of $\lambda-T$ is non-zero is contained in $\sigma_{o}(T) \backslash \sigma_{o e}(T)$.

\section{REFERENCES}

1. B. Aupetit, Inessential elements in Banach algebras, Bull. London Math. Soc. 18 (1986), 493-497.

2. W. Arendt, On the o-spectrum of regular operators and the spectrum of measures, Math. Z. 178 (1981), 271-287.

3. W. Arendt and D. R. Hart, The spectrum of quasi-invertible disjointness preserving operators, J. Functional Analysis, 68 (1986), 149-167.

4. W. Arendt and A. R. Sourour, Perturbation of regular operators and the order essential spectrum, Indag. Math. 89 (1986), 109-122.

5. B. A. Barnes, Inverse closed subalgebras and Fredholm theory, Proc. Roy. Irish Acad. Sect A 83 (1983), 217-224. 
6. B. A. Barnes, G. J. Murphy, M. R. F. Smyth and T. T. West, Riesz and Fredholm theory in Banach algebras, (Pitman, 1982).

7. F. F. Bonsall and J. Duncan, Complete normed algebras, (Springer-Verlag, 1973).

8. H. G. Dales and W. H. Woodin, An introduction to independence for analysts, London Math. Soc. Lecture Notes 115, (Cambridge University Press, 1987).

9. L. Groenewald, R. E. Harte and H. Raubenheimer, Perturbation by inessential and Riesz elements, Quaestiones Math., 12 (1989), 439-446.

10. R. E. Harte, Fredholm theory relative to a Banach algebra homomorphism, Math. Z., 179 (1982), 431-436.

11. J. G. Hocking and G. S. Young, Topology (Addison Wesley, 1961).

12. H. Raubenheimer, The o-spectrum of r-asymptotically quasi-finite rank operators, Quaestiones Math., 7 (1984), 299-303.

13. $\mathrm{H}$. Raubenheimer, $r$-Asymptotically quasi-finite rank operators and the spectrum of measures, Quaestiones Math., 10 (1986), 97-111. $79-84$.

14. H. H. Schaefer, On the o-spectrum of order bounded operators, Math. Z., 154 (1977),

15. A. R. Schep, Positive diagonal and triangular operators. J. Operator Theory, 3 (1980), $165-178$.

Department of Mathematics and Applied

Mathematics

Potchefstroom University for Christian Higher Education

POTCHEFSTROOM 2520

SOUTH AFrica
Department of Mathematics

University of the Orange Free State BLOEMFONTEIN 9300

South Africa 